\title{
Technological Dimensions of Nearly ZEB Design: Evolving toward a Nearly Zero Energy Oriented Landscape
}

\author{
Filippo Angelucci*
}

\author{
Department of Architecture, University of Chieti-Pescara, “G. d’Annunzio”, Viale Pindaro, 42 - 65127 Pescara, \\ Italy
}

\begin{abstract}
Today, the implementation framework of Zero Energy Building strategies is characterised by a complex transitional phase. In fact, it is still difficult to achieve completely autonomous buildings, disconnected from any power-
\end{abstract} supply network.

Despite the negative impact of climate-change and the progressive loss of non-renewable resources on our lifestyles, the global economic-financial crisis, local cultural-technological barriers, and the cost/complexity of design processes keep investment in this area unattractive.

However, there is an intermediate approach that can facilitate a gradual re-direction of building actions through the ZEB logic. It can be identified in the alternative of Near Zero Energy Building (Nearly ZEB or Near Net ZEB).

The Nearly ZEB approach, with its multiplicity of design aspects (i.e. cognitive, analytical, technical, and managerial) may configure a widespread state of progressive transition towards the architectural/inhabitable constructions sought by the 20-20-20 logic, at the scale of the building, the city, and the landscape.

These constructions have lower emissions, produce more energy from renewable sources, consume less non-renewable energy, and can "also" reach the objective of total energy autonomy (ZEB) or Plus Energy.

A twofold operating scenario emerges from this point of view. It is centred on the technological dimensions of designing a Nearly Zero Energy living space by overcoming the traditional concept of a building as a single object.

On the one hand, there is a need for a greater interaction between technological innovations and inhabitable spaces, in a trans-scalar key; design becomes an open process of technological-environmental modifications that addresses the transition towards the status of ZEB.

On the another hand, it becomes fundamental that the relationship between interior and exterior space, both public and private, is increasingly focused on the design of interface-systems in order to harmonise three new levels of relations (city-building, city-land, and building-land) and to configure a Nearly Zero Energy Oriented Landscape.

These aspects emerge from the contributions presented in this special issue on The Technological Dimensions of Nearly Zero Energy Building Design and will be addressed in this essay.

Keywords: Nearly ZEB Process, Environmental-Technological Design, Inter-systemic/Trans-scalar Design, Environmental Interfaces, Nearly Zero Energy Oriented Landscape.

\section{FROM ZEB TO NEARLY ZEB}

The concept of Zero Energy Building (ZEB) embraces multiple definitions that imply radical modifications to the process of designing a building and to the approaches, methods and tools employed in the configuring the morphology of inhabitable spaces. In fact, a ZEB, to merit this label, does not simply presuppose the use of renewable energies, but also the possibility that the building is totally autonomous from any energy networks. This condition of total autonomy has led, above all, to a commercial use of the term ZEB. In reality, reflections on the different experiments carried out in the search for total energy independence since the 1960s (i.e. Passivehaus, Zero Energy Home, Maison autonome en énergie, Green

*Address correspondence to this author at the Department of Architecture, University of Chieti-Pescara, "G. d'Annunzio", Viale Pindaro, 42 - 65127 Pescara, Italy; Tel: +39.085.45.37.332; E-mail: filippo.angelucci@unich.it, filangel@alice.it
Building, Heliotrop) outlined a much more complex framework for the creation of ZEBs. An operative framework that offers common strategies of works, though with notable differences in their implementation owing to the different socio-economic, territorial, and cultural conditions tied to specific sites of intervention [1].

As a result, it is necessary to consider a vaster balancing of different resources - energy-related, technological, economic, and infrastructural - and the introduction of a more complete definition of the Net Zero Energy Building (Net ZEB) that includes the possibility to go off-grid and function in complete energy autonomy.

The Net ZEB concept emphasises the condition of balancing energy consumed with energy produced over a period that is generally equivalent to one year [2]. A more specific description of ZEBs was specified by the NREL/National Renewable Energy Laboratory in relation to the limits of building systems and flows of 
energy produced/consumed by identifying four principal classes of Net ZEBs (Net Zero Site Energy, Net Zero Source Energy, Net Zero Energy Costs and Net Zero Energy Emissions) [3].

All the same, a complete implementation of Net ZEB strategies remains difficult, above all during the current phase of a general economic-financial recession that renders long-term investments in environmental sustainability less attractive and riskfree. This has led to the development of alternative lines of intervention that serve to frame construction within a perspective aimed at gradually moving toward ZEB status. One result is the definition of Near Net Zero Energy [4] developed from the European Directives 2010/31/EU on the "Energy Performance of Buildings" (article 17) and 2012/27/EU on "Energy Efficiency" which introduce the concept of nearly zero energy.

The logic of nearly zero energy, as a system of coordinated actions designed to confront energy crises, does not stop at the results of building design. Advancing further, it brings into play the multiple definitions of design (cognitive, analytical, technical, implementation, managerial) that may help configure a more widespread progressive transition toward the inhabitable built, urban, and territorial constructions pursued by $20-20-20$ strategies ${ }^{1}$.

For design, nearly zero energy strategies thus assume the specific connotation of an open and ongoing process. The concept of nearly Zero Energy (nZE) design, other than reducing energy consumption and polluting emissions and increasing energy production from renewable sources, requires the adoption of a broader and more complex convergence between material/immaterial innovation aspects which are characteristic of the building processes employed in the production, transformation and consumption of energy in the human habitat.

In this sense, design takes on an a-scalar, or better yet trans-scalar connotation, as it is required to oscillate between the feedbacks provided by various levels of intervention [5]. By forecasting a radical change in construction through the involvement of

\footnotetext{
${ }^{1}$ For example, in the European Community, the goal of 20-20-20 established with the Directive 2009/28/EC $\left(-20 \% \mathrm{CO}_{2}\right.$ emissions, $+20 \%$ renewable energy, $+20 \%$ energy efficiency) are already a very challenge for ZEB strategies. These goals are oriented towards a much more ambitious levels: $-40 \% \mathrm{CO}_{2}$ emissions, $+27 \%$ renewable energy, $+27 \%$ energy efficiency to be achieved by 2030 (Paris CIP21 meeting 2015) and rising to $+30 \%$ of energy efficiency by 2030 (proposal COM(2016) 761 for the variation of Directive 2012/27/EU).
}

disciplines not exclusive to the energy sector, but also to environmental, transportation, information and communications technologies, nZE design focuses on multiple technological dimensions able to advance a new culture of dwelling in which users/communities become active and responsible inhabitants/consumers/ producers of energy.

This role, focused on a renewed set of relations that make local communities accountable for the overall energy needs of society may become the principal driver of changes to the built environment. Passing from the traditional notion of isolated and energyintensive constructions to the construction of complex inhabitable organisms that interact with intelligent networks of building, urban and territorial components may gradually lead to the development of an architecture based on passive technologies and positive energy performance.

This is an idea of Architecture in constant evolution, which may "also" arrive at a final condition of total energy autonomy (ZEB) or of Plus Energy.

In operative terms, the resulting framework is twofold and concerns specifically technological aspects.

On the one hand the need for increased interaction between technological innovations in the energy sector and inhabitable spaces. We must reconsider nZE design as a process of technological-environmental modification that remains open and on going, in order to manage the transition toward ZEB status.

For other aspects, it is fundamental that relationships between interior/exterior space, public/ private, open/closed, focus increasingly more on the design of technological-environmental interfaces, ready to configure a Nearly Zero Energy Oriented Landscape, working with at least three levels of relation: the interface between building-city, between city-land and between building-land.

\section{THE CENTRAL ROLE OF TECHNOLOGICAL DISCIPLINES IN nZE DESIGN}

A nearly zero energy design philosophy reveals the need to configure the future built environment as a system that is connoted by technological choices for transforming the various forms of energy present in the territory.

In this sense, the nZE design process operates at different levels: the participatory involvement of 
inhabitants, the critical analysis of the needs of end users, a reading of the restrictions imposed by nature, legislation and economic conditions, the impacts on local cultures of global technological solutions, the processes transforming systems of dwelling/settlement, including spontaneous examples, induced by changing needs and behaviours.

During the early season of architectural experiments conducted between the Kyoto Protocol (1997) and the Copenhagen Conference (2009), the challenge of energy sustainability could be framed by two principal macro-objectives for design: the acceptability of technological innovations by users and local communities and the correlation between these innovations and specific territorial variables.

Energy sustainable design assumed a specifically systems-oriented, interdisciplinary and integrated definition (i.e., the integration of global innovations within local building cultures, the integration of specific disciplinary specifications), moving along strategic lines founded on the communicability of innovations, local energy identities and energy communities. In the extreme, this integration was founded also on maximizing energy performance through hyperfuturistic visions. Technological design managed to forecast truly technological energy landscapes [6] with high/very high levels of performance that hinted at the ease of achieving the objectives of ZEB.

However, the highly unstable socio-economic situation that spread across the globe in 2008 raised other questions. The operational limit of approaches based on an attention focused exclusively on qualitative, aesthetic, and formal components was opposed to an equally narrow idea of guaranteeing an increase in energy sustainability by working exclusively with numeric and quantitative data.

At a time marked by the scarcity of physical and economic resources, the dichotomy quality/quantity is no longer sufficient for responding to the energy needs expressed by the territory and its inhabitants. The binomial acceptability/correlation must now accompany two other macro-objectives in order to best describe the transitory conditions of contemporary societies, including adaptability (of users/inhabitants to economic, social and climate change) and sharing (of energy, physical and spatial resources).

These objectives centre design on the transitory nature of times, spaces, practices and uses. Hence, there is a need for design to assume a new, intersystemic, trans-disciplinary and interactive characterisation, as the flow of technological-environmental projects that serve to re-establish symbiotic processes of adaptation (chancing our lifestyle) to a new condition marked by a scarcity of resources [7].

Therefore, nZE design must work to recompose the multiple identities, qualities, and variables of the energy question within a more general perspective of modifying habitat in the search for new synergies between people, territory, and energy resources. This re-composition of qualitative and quantitative aspects, global and local, presupposes that buildings, cities, and territories are part of an adaptable inhabitable system. A system that is reactive to the changing conditions of the external environment and the behaviour of its inhabitants, which interacts with hard infrastructural networks to procure energy, but also with smart and light infrastructures.

\section{TOWARD A NEARLY ZERO ENERGY ORIENTED LANDSCAPE (nZEOL)}

Within the perspective of a nearly Zero Energy Design, the energy sustainable human habitat of the future once again assumes the significance of a point of convergence between the natural and anthropic factors used to constantly reproduce ecosystemic, dynamic, and changing equilibriums.

If the experiments conducted to date with Net ZEB describe the technological scenarios of an energy landscape focused on increasingly higher performance levels and under tendentially stable environmental conditions, the scenarios of a nearly Zero Energy logic are entirely different.

In fact, there is a need to move from a nearly Zero Energy Oriented Landscape, which incorporates environmental and performance instabilities (climatic, economic, social and energy) in order to transform it into integral parts of an open project for the built environment, designed to care for, conserve and transform the multiple dimensions of energy tied to dwelling.

Designing a nearly Zero Energy Oriented Landscape signifies constructing a system of relations that is not concentrated on a product, but centred on understanding processes of energy exchange which become generators of the structures and forms of settlements at the scale of buildings, cities and territories. 
These processes are unstable, due to the technical difficulties of producing/conserving energy obtained from renewable sources, the heterogeneous distribution of non-fossil energy resources, and the elevated costs of technological innovations. Thus, there is a need to confront the design of future nZEOL as a project focused on the adaptation of inhabitants and settlements to the restrictions induced by the transition toward an economy of renewable energies that must tend to reduce emissions and consumption, but also to increase the sustainable production of energy. The principal restrictions charactering the passage toward an nZEOL include: the fluctuation of renewable energies, the low productivity of renewable energies with respect to fossil fuels, the lost capacity of individuals/societies to optimise their activities in relation to the availability of energy [8].

This new operative condition, characterised by the scarcity, variability, and discontinuity of renewable energies has a particular effect on the process of achieving a zero energy state.

To use the words of Enzo Tiezzi, there is a need to privilege the adoption of logics of intervention in the built environment based on increasing "negentropy" in order to build harmonic relations between buildings, systems, cities, territories, biodiversity and complexity, and return to learning how to capture and use energy in its various forms [9].

The nearly Zero Energy Oriented Landscape is thus configured as a hybrid "system of systems": based on the wise and reasonable utilisation of traditional and innovative technologies of energy use; projected toward an organic development compatible with the needs of local realties; attentive toward the framework of environmental responsibilities of the global energy market.

While this landscape is undoubtedly technological, it is based on multiple dimensions of innovation tied to diverse approaches (i.e., lowering consumption, maximizing interactions, reducing grey energy, aiding self-production, facilitating the sharing of energy, favouring a culture of evaluation) [10] in order to reestablish different levels of co-evolution between energy, nature and technologies [11].

\section{THE TECHNOLOGICAL DIMENSIONS OF nZEOL}

Designing according to the logic of nZE presupposes a vaster project of "construction" that moves beyond the physical limits of a building, the semiotic confines of the city and its infrastructures and, the administrative borders of the territory.

This signifies utilizing natural/technological resources and the spaces in a more balanced manner, passing progressively from an energy-intensive vision of construction, to an energy-economic mode that tends toward an energy-producing way of building.

Designing beyond the boundary of buildings, cities, and territories means considering physical elements not as limits, barriers, or perimeters, but instead as thresholds of exchanges. It signifies focusing nZE design toward a reinterpretation of physical limits as systems of technological-environmental interface.

These systems of interface should favour [12]:

- at the land level, the constitution of energy networks, communities and districts, balanced land uses, building densities and different typologies of sustainable mobility;

- at the urban level, the use of mixed energy supply-chains, micro-climate control (temperature, ventilation, solar radiation, water), the correct use of open and green spaces;

- at the building level, the minimisation of the negative effects caused by the needs of heating, cooling, energy supply and the adaptation of buildings rather than their demolition/substitution.

Only in these terms is it possible to gradually contribute to the "construction" of a nearly Zero Energy Oriented Landscape, in which it is correct to speak of systemic levels and not of scales. In fact, each of these systems embraces multiple scales of intervention with at least three technological dimensions of interface: building-city, city-land and building-land.

Building-city technological interfaces presuppose the use of technological and natural resources that reinterpret the building envelope no longer as a physical barrier, but as an osmotic membrane, that surpasses the clear separation between the interior spaces of the building and the exterior space of the city. Viewed in this manner, inhabitable space, building envelope and city constitute a unique macro-system marked by the open and adaptive interaction between the physical elements of a building and the city (spaces, building systems, equipment, users) and the immaterial and fluid components of the built environment (air, water, landscaping, flows of users, traffic, waste, services). 
Energy variables are no longer tied exclusively to thermodynamic issues. The macro-system of the building-city interface tends to: construct coherent relations between interior space and exterior environmental dynamics, control flows of materials, information and energy passing through buildings, gardens and neighbourhoods and configure surfaces/volumes to host uses and modifications, even those that succeed one another over time [13].

Technological city-land interfaces seek to reposition energy issues within a process of constructing a habitat, inevitably destined to unfold in different phases of evolution. It acts as a regulating apparatus for energy relations involving the city/land to favour, over time, a capacity for the built environment to evolve in its coherent response to the ever faster and increasingly more unpredictable shifts in climatic, political, administrative, economic, ecological, and social structures. The macro-system of the city-land interface intervenes to permit the implementation of actions that favour the adaptation of space and its inhabitants to a state of zero energy: at the level of infrastructure, through physical interventions on in-between/residual spaces, to support a reduction in energy consumption; at the instrumental level, through immaterial actions designed to infuse processes of inhabitation with codes of behaviour and virtuous practices of adapting energy uses to variations in available resources.

Technological building-land interfaces constitute another dimension of intervention aimed at the reorganisation of the human habitat and its dynamics of using/producing energy in trans-scalar terms. They develop primarily through the spread of ICT and interactive networks, but also through the miniaturisation of technologies for producing energy from renewable resources. This macro-system of interface has a twofold identity. As an immaterial entity, on line, it facilitates the constitution of a responsible and virtuous community of inhabitants who adapt to sharing spaces, energy resources, and ideas/solutions for improving the energy performance of buildings, cities and the land. As a system of material interventions, on site, that contribute to reinterpreting energy relations between building-land through the constitution of localised/widespread micro-generation networks from RES (energy islands, mini-networks, clusters), in order to gradually break free of the unified/centralised model of distribution and move toward Net ZEB and Plus Energy objectives [14].

\section{CONCLUSIONS}

The contributions presented for the Technological Dimensions of Nearly Zero Energy Building Design call are a testimonial to current experimental research into the problems of nZE within a more general reorientation of the processes of designing the built environment. The essays presented in this volume are undoubtedly only part of a much vaster framework unfolding at the international level in relation to Nearly Zero Energy building. It is however important to point out that all of their authors belong to a research cluster dedicated specifically to this topic: the nZEB (http://www.sitda.net/index.php/cluster/nzeb.html), instituted in Italy as part of the activities of the SITdA Società Italiana della Tecnologia dell'Architettura (http://www.sitda.net/index.php).

The texts that follow cannot summarize the broader scientific activities developed by the investigating community of the nZEB/SITdA cluster, but they make it possible to retrace the themes emerging from an $\mathrm{nZE}$ design vision and the relative principal challenges to be confronted in the future.

In the essay "The future of research and experimentation in technological design of the relationship between energy, architecture, environment", Fabrizio Tucci proposes a reflection on the strategic importance of research to innovation in the sector of nZEB policies. He proposes an a-scalar vision open toward the convergence between the activities of the nZEB cluster and those of the national group "Green Economy for Architecture and Cities" which he coordinates at the SITdA.

Chiara Tonelli and Ilaria Montella, in "Simulation of dynamic thermal behaviour for housing in warm climate: open scientific problems", emphasise the contribution made by the mass of building envelopes to the thermal regulation of inhabited spaces in Mediterranean climates, focusing attention on an innovative reconsideration of local and traditional building techniques.

In "Adaptive models for the energy efficiency of building envelopes", Martino Milardi focuses on the centrality of the capacity for building envelopes to adapt, to configure dwelling systems able to react to variations in the exterior environment and establish synergetic relations that differ from case to case, with local context. 
The essay "Form follows Zero Energy. Technological Design for Sustainable Housing in Extreme Climate Areas", by Di Sivo, Basti, Ladiana and Cellucci, explores the issue of dwelling in extreme climates, identifying the wise use of water and solar resources as central to the establishment of a continuity between tradition and innovation.

Finally, the contribution by Elisa leie "Energy efficiency of low quality built heritage: methodological reflections on achieving the NZEB through a case study in the city of L'Aquila" looks at the reuse of low quality buildings that, through the rehabilitation and improved energy performance, providing opportunities for re-establishing functional and formal relations with the urban context.

All these essays are evidence of an Italian culture of technological design that looks at both technical and natural resources in a balanced and relational manner; without slipping into the easy temptations of top-down innovation or innovation at all costs, though neither adopting a refusal of innovation and change.

They are the expression of experiments underway in Italy tied to issues of designing and building for Nearly Zero Energy that, however, as demonstrated also by the recurring attention to Mediterranean areas and extreme climates, face up to broader and extrageographical issues.

Issues that include present-day climate changes at the global/continental level and the inevitable changes they comport - today and in the future - for society and lifestyles, anticipating the challenges raised by necessary changes to the way we approach design.

\section{REFERENCES}

[1] Filippi M, Fabrizio E. II concetto di zero energy building. In: Verso gli edifici a "energia quasi zero": le tecnologie disponibili. Atti del convegno. Milano: AICARR 2011; pp. 1-14.
[2] Sartori I, Napolitano A, Marszal AJ, Pless S, Torcellini P, Voss K. Criteria for definition of net zero energy buildings. In: Proceedings of EuroSun 2010: International conference on solar heating, cooling and buildings: 28 September - 1 October 2010. Graz: EuroSun 2010. https://doi.org/10.18086/eurosun.2010.06.21

[3] Torcellini P, Pless S, Deru M, Crawley D. Zero energy buildings: A critical look at the definition. NREL/National Renewable Energy Laboratory and Department of Energy. Springfield: NREL 2006. Available from: https://www.nrel.gov/docs/fy06osti/39833.pdf.

[4] Hootman T. Net zero energy design. Hoboken: John Wiley and Sons 2013; pp. 1-25.

[5] Tucci F. Design, technology, energy: quality of building and living in nearly zero energy and zero emission architecture. In: Lucarelli MT, Mussinelli E, Trombetta C, Eds. Cluster in progress. The architectural technology network for innovation. Rimini: Maggioli Editore 2016; p. 75.

[6] Angelucci F. editor. La costruzione del paesaggio energetico. Milano: Franco Angeli 2011; pp. 17-48, 65-75.

[7] Friedman Y. L'architecture de survie: Une philosophie de la pauvreté. Paris: Éditions de l'éclat 2003; pp. 59-97.

[8] Stremke S. Designing sustainable energy landscapes. Concepts, principles and procedures. $\mathrm{PhD}$ Thesis, Wageningen: Wageningen University 2010; pp. 75-96.

[9] Tiezzi E. Neghentropy against destruction. In: Heimdall's horn. Citizenship of the earth - A new alliance between peoples, ideas and cultures. Proceedings of XXVIII Pio Manzu International Conference. Rimini: Pio Manzù Research Centre 2002; pp. 111-117.

[10] Tucci F. Design, technology, energy: Quality of building and living in nearly zero energy and zero emission architecture. In: Lucarelli MT, Mussinelli E, Trombetta C, Eds. Cluster in progress. The architectural technology network for innovation. Rimini: Maggioli Editore 2016; pp. 76.

[11] Mulder K. The technological landscape. In: Sijmons D, Hugtenburg J, van Hoorn A, Feddes F, Eds. Landscape and energy. Designing transition. Rotterdam: Nai010 publishers 2014; pp. 368-380.

[12] Vivienne Brophy V, Owen Lewis J. A green Vitruvius. Principles and practice of sustainable architectural design, 2nd ed. London: Earthscan Edition 2011; pp. 3-43.

[13] Habraken NJ. The structure of the ordinary. London: Teicher 1998.

[14] Habash G, Chapotchkine D, Fisher P, Rancourt A, Habash $\mathrm{R}$, Norris W. Sustainable design of a nearly zero energy building facilitated by a smart microgrid. Journal of renewable energy; Volume 2014 (2014), Cairo: Hindawi Publishing Corporation 2014. Available from: https://www.hindawi.com/journals/jre/2014/725850/.

(C) 2017 Filippo Angelucci; Licensee Lifescience Global.

This is an open access article licensed under the terms of the Creative Commons Attribution Non-Commercial License (http://creativecommons.org/licenses/by-nc/3.0/) which permits unrestricted, non-commercial use, distribution and reproduction in any medium, provided the work is properly cited. 